\title{
ANALYTICPOS: FERRAMENTA PARA ANÁLISE DO PERFIL ACADÊMICO DOS CURSOS DE PÓS-GRADUAÇÃO NO INSTITUTO FEDERAL CATARINENSE - CAMPUS DE VIDEIRA
}

http://dx.doi.org/10.5902/2318133819813

\author{
Leila Lisiane Rossi \\ Instituto Federal Catarinense - campus de Videira, Brasil. \\ Luiz Gustavo Moro Senko \\ Instituto Federal Catarinense - campus de Brusque, Brasil. \\ Wanderson Rigo \\ Instituto Federal Catarinense - campus de Videira, Brasil. \\ Jeferson José Schneider Boesing \\ Instituto Federal Catarinense - campus de Videira, Brasil.
}

\begin{abstract}
Resumo
Neste texto apresenta-se a ferramenta Analyticpos, desenvolvida em um projeto de pesquisa em andamento e que tem como objetivo permitir a análise multidimensional dos dados dos estudantes dos cursos de pós-graduação do Instituto Federal Catarinense - IFC - campus de Videira. A ferramenta foi desenvolvida usando software livre, conforme recomendações do governo federal, e tem como propósito auxiliar os coordenadores na tomada de decisão e na adoção de políticas educacionais para os cursos. O módulo de pós-graduação foi desenvolvido como continuidade de outro projeto, cujo objetivo foi o de avaliar os cursos superiores do IFC - campus de Videira por meio de uma ferramenta similar.

Palavras-chave: data webhouse, mineração de dados, educação.
\end{abstract}

\section{ANALYTICPOS: TOOL FOR ACADEMIC PROFILE ANALYZIS OF THE POSTGRADUATE COURSES OF THE INSTITUTO FEDERAL CATARINENSE - CAMPUS DE VIDEIRA}

\section{Abstract}

This paper presents the Analyticpos tool developed in a research project in progress and is aimed to allow the multidimensional data analyze of the postgraduate academic of Federal Institute of 
Santa Catarina - campus de Videira. The Analyticpos was developed using free software according to federal government regulations and is aimed to help the coordinators in the decision-making process and in adopting educational politics for the courses. The postgraduate tool is a module of the research project that is being developed to continuous another one similar about graduate courses in the same institution.

Key-words: data webhouse, data mining, education. 


\section{Introdução}

dentificar e conhecer o perfil dos estudantes nas instituições de ensino é uma tarefa imprescindível para os administradores. Pelo perfil é possível tomar decisões e adotar as políticas necessárias. No âmbito dos cursos de pósgraduação, que no campus de Videira iniciaram em 2011, esta ação foi entendida como forma de prever a continuidade dos mesmos.

Com o gradual aumento no volume de dados considera-se importante o uso de ferramentas automatizadas para facilitar a manipulação dos mesmos, a análise e o processo de tomada de decisão. As ferramentas analíticas de data webhouse - armazém de dados na web - e de data mining - mineração de dados - facilitam este processo.

Com o objetivo de facilitar a avaliação e a análise do perfil dos estudantes dos cursos de pós-graduação do Instituto Federal Catarinense - campus de Videira, foram desenvolvidos alguns testes com o uso a ferramenta Analyticpos. Pelos testes realizados foi possível conhecer e analisar o perfil dos estudantes e identificar, por exemplo, a proveniência, os motivos da evasão nos cursos ou associar os que estão regularmente matriculados e em andamento. A ferramenta Analyticpos permitiu a realização de consultas analíticas online - Olap (Inmon, 1996) na web pela visualização multidimensional dos dados. Esta visualização, por sua vez, se constitui numa ferramenta que auxilia os coordenadores dos cursos e a gestão no processo de análise do perfil acadêmico e na tomada de decisão no que diz respeito às demandas dos cursos.

\section{Cursos de pós-graduação}

O IFC - campus de Videira mantém três cursos de pós-graduação lato sensu. Os cursos são Desenvolvimento Web, Agronegócios e Educação. $O$ curso de Desenvolvimento Web teve duas turmas até o momento, ambas com entrada em 2011. O curso de Agronegócios também teve duas turmas, sendo uma delas com entrada em 2011 e a outra com entrada no ano de 2013. E o curso de Educação teve somente uma turma, com ingresso em 2011. Todos os cursos são oferecidos conforme disponibilidade da instituição e público interessado.

\section{Ferramentas similares e a Analyticpos}

O Wead é um data webhouse desenvolvido em um projeto de pesquisa na Universidade Federal de Mato Grosso do Sul (Turine, 2006) que auxilia os coordenadores de ensino a distância na tomada de decisão com base no perfil dos estudantes dessa modalidade de ensino.

O Wead (Turine, 2006) foi desenvolvido com o uso de software livre, como Java (2015) para a programação, Pentaho BI Server (Pentaho, 2015) como servidor/interpretador dos dados e PostgreSQL (Postgresql, 2015) para o armazenamento.

A ferramenta DWSI (Campelo, 2009) também foi desenvolvida pela UFMS e teve como objetivo avaliar o perfil de um único curso presencial: Sistemas de Informação.

A ferramenta Analytic, por sua vez, (Rossi et. al., 2015a; Rossi et. al., 2015b) foi desenvolvida no IFC - campus de Videira e tem como objetivo avaliar o perfil acadêmico dos cursos superiores. 
O presente trabalho está sendo desenvolvido no IFC campus de Videira e é direcionado aos cursos de pós-graduação, os quais são, até o presente momento, presenciais. A ferramenta analítica usada foi o Pentaho bi server para o data webhouse.

Desenvolveu-se, inicialmente, um formulário para a entrada de dados a partir do cadastro dos estudantes na secretaria acadêmica. Com base neste formulário as informações foram carregadas para a base de dados. Na sequência, o modelo Multidimensional Estrela (Kimball, 2002) foi escolhido para a criação do cubo, sendo idêntico ao usado nos projetos anteriores para a graduação permitindo, assim, a disponibilização dos dados em uma tabela Fato, centralizada com as medidas necessárias para a realização das consultas Olap e várias tabelas Dimensão ligadas a ela.

O cubo Analyticpos possui as seguintes tabelas Dimensão: sexo, estado civil, portador de diploma, pública ou particular, situação-curso, forma de entrada no curso, ano de entrada, curso. A tabela Fato apresenta as medidas necessárias para a realização das consultas Olap, tais como a quantidade de estudantes que ingressam no curso em um determinado ano, a quantidade de estudantes de cada sexo. A partir do modelo multidimensional armazenado no PostgreSQL (Postgresql, 2015), foi gerado o cubo XML Extensible markup language - usando a ferramenta Schema Workbench (Pentaho, 2015). O cubo XML é necessário para a criação das consultas Olap pela ferramenta Pentaho BI Server (Pentaho, 2015), a qual possibilita a visualização dos dados mutidimensionais na web. Estas ferramentas foram escolhidas pela familiaridade da equipe em trabalhar com elas em projetos anteriores, além do fato das mesmas serem software livre.

A consulta Olap é realizada com auxílio da linguagem de manipulação de dados MDX - Multidimensional expressions. Trata-se de uma linguagem semelhante ao SQL Structured Query Language -, porém utilizada especificamente em data webhouse. É possível utilizar a linguagem SQL para manipular o cubo, entretanto, a mesma torna-se mais complexa se comparada à sua versão equivalente em MDX.

A figura 1 a seguir apresenta a tela de login do Data Webhouse. 
Figura 1 -

Tela de login.

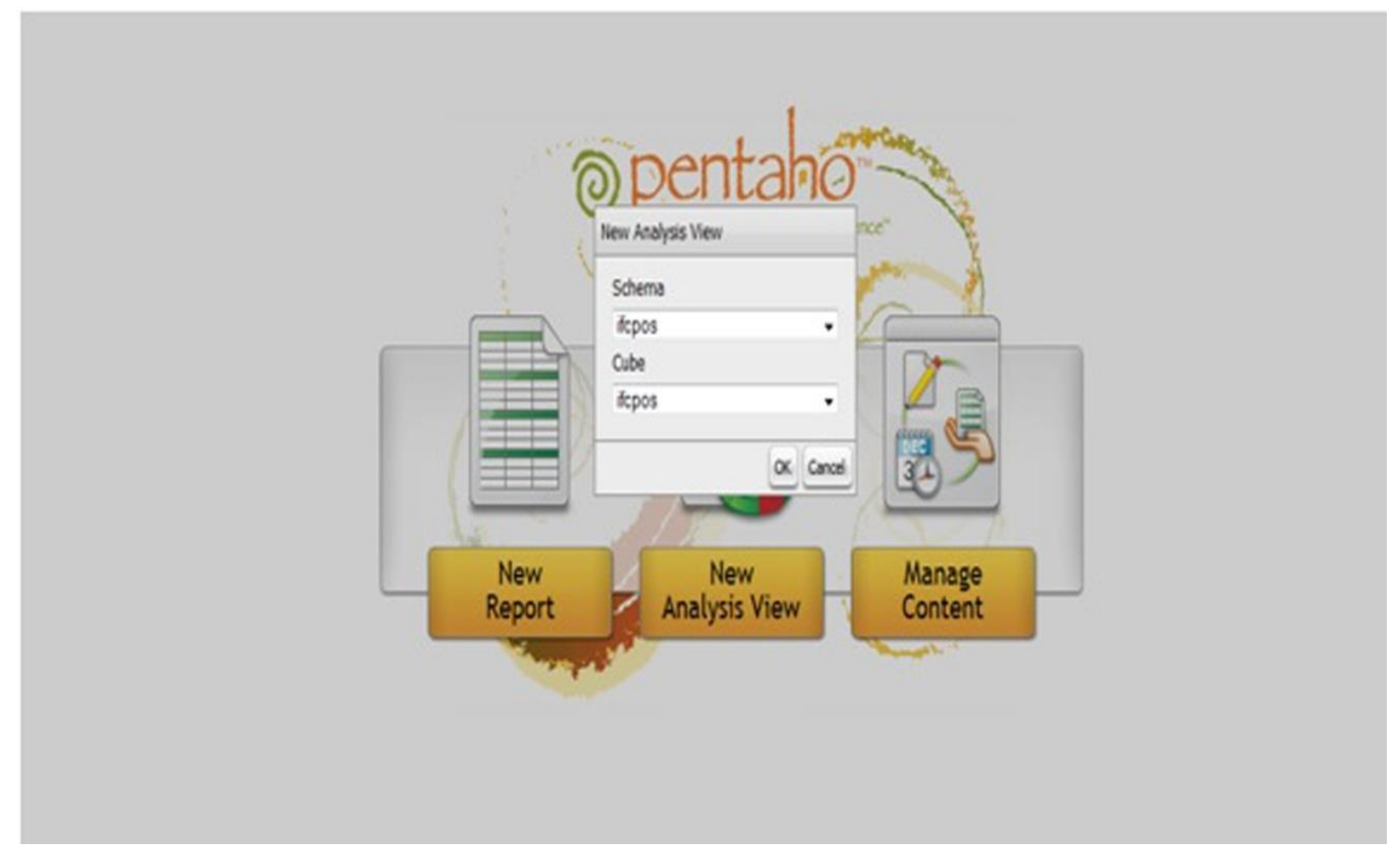

As consultas dinâmicas habilitam a navegação drill-down-up, ou seja, o usuário pode escolher o nível de detalhamento das consultas como, por exemplo, o total de estudantes por situação no curso.

\section{Resultados obtidos}

Como resultado criou-se um data webhouse pelo qual é possível conhecer e analisar o perfil acadêmico dos cursos de pós-graduação do IFC - campus de Videira. A figura 2 mostra a proveniência dos estudantes em relação ao ensino superior: a maioria estudou em escola particular. Vale ressaltar que nenhum dos cursos de pós-graduação do IFC campus Videira possui estudantes do próprio campus, considerando que não foi formada nenhuma turma nos cursos de graduação até o momento. 
Figura 2 -

Consulta Olap Analyticpos - proveniência de estudantes.

\begin{tabular}{|c|c|c|c|c|c|c|c|c|}
\hline \multicolumn{9}{|c|}{ New Analyss View Q } \\
\hline \multicolumn{9}{|c|}{ 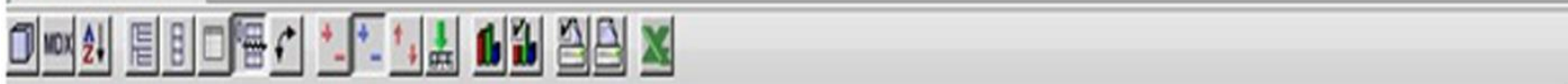 } \\
\hline & & & & & & & & Nezsures \\
\hline sex & estodo_owl & portador_diploma & publica_particular & stuacao_ourso & forma_entrod__arso & an_entroda & curso & - total \\
\hline \multirow[t]{8}{*}{${ }^{7}$ All sexos } & \multirow[t]{8}{*}{${ }^{7}$ All estado_ovils } & \multirow[t]{8}{*}{${ }^{\dagger}$ All portador_diplomas } & EAl pudica_particulars & ${ }^{7}$ All sturaco__ursos & ${ }^{-}$All forma_entrada_oursos & ${ }^{\top}$ All ano_entradas & ${ }^{\oplus}$ All cursos & 176 \\
\hline & & & \multirow[t]{4}{*}{ PARTICUAR } & \multirow[t]{4}{*}{${ }^{\dagger}$ All sturceo_arsos } & \multirow[t]{4}{*}{${ }^{\dagger}$ All forma_entrodo_arsos } & \multirow[t]{4}{*}{${ }^{\dagger}$ All ano_entradas } & EAll arsos & 150 \\
\hline & & & & & & & AGRONEGOCODS & 54 \\
\hline & & & & & & & EOUCACAO & 30 \\
\hline & & & & & & & DFORMATICA & 66 \\
\hline & & & \multirow[t]{3}{*}{ PUBLCA OUTRA } & \multirow[t]{3}{*}{${ }^{7}$ All sturace_oursos } & \multirow[t]{3}{*}{ (T) All forma_entrodz_arses } & \multirow[t]{3}{*}{ 9All ano_entrodas } & $\Xi_{\text {All oursos }}$ & 26 \\
\hline & & & & & & & AGRONEGOCOOS & 17 \\
\hline & & & & & & & DFORMATICA & 9 \\
\hline
\end{tabular}

Figura 3 -

Consulta Olap Analyticpos - estado civil.

\begin{tabular}{|c|c|c|c|c|c|c|c|c|}
\hline \multicolumn{9}{|c|}{ New Analysis View 8} \\
\hline \multicolumn{9}{|c|}{ 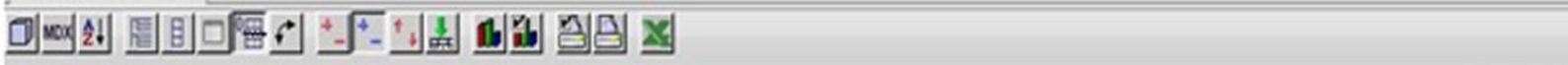 } \\
\hline & & & & & & & & Measures \\
\hline $\sec 0$ & estado_ovil & portador_diploms & publica_perticular & stuaceso_curso & forma_entrods_curso & ano_entrods & curso & - total \\
\hline \multirow[t]{17}{*}{$\theta_{\text {All sexos }}$} & EAll estado_ovls & - All portador_dplomas & - All publica_partioulars & ${ }^{+}$All stuaceso_cursos & ${ }^{+}$All forma_entrada_cursos & (All ano_entrodas & ${ }^{\oplus}$ All cursos & 176 \\
\hline & \multirow[t]{4}{*}{ AMASIADO } & \multirow[t]{4}{*}{ - All portador_diplomas } & \multirow[t]{4}{*}{ - All publica_particulars } & \multirow[t]{4}{*}{ ¿All stuaceo_cursos } & \multirow[t]{4}{*}{ ¿All forma_entroda_cursos } & \multirow[t]{4}{*}{ - All ano_entrodas } & All cursos & 12 \\
\hline & & & & & & & AGRONEGOCLOS & 6 \\
\hline & & & & & & & EDUCACAO & 5 \\
\hline & & & & & & & DNFORMATICA & 1 \\
\hline & \multirow[t]{4}{*}{ CASADO } & \multirow[t]{4}{*}{ ๑Al portador_diplomas } & \multirow[t]{4}{*}{ - All publica_darticulars } & \multirow[t]{4}{*}{ TAll stuacso_cursos } & \multirow[t]{4}{*}{ † All forma_entrade_cursos } & \multirow[t]{4}{*}{ TAll ano_entrados } & $\nabla_{\text {All cursos }}$ & 45 \\
\hline & & & & & & & AGRONEGOCIOS & 18 \\
\hline & & & & & & & EDUCACAO & 12 \\
\hline & & & & & & & DNFORMATICA & 15 \\
\hline & \multirow[t]{4}{*}{ SEPARADO } & \multirow[t]{4}{*}{-All portador_diplomas } & \multirow[t]{4}{*}{-All publica_particulars } & \multirow[t]{4}{*}{ (1) All stuaceo_cursos } & \multirow[t]{4}{*}{ †All forma_entrada_cursos } & \multirow[t]{4}{*}{ †. All ano_entradas } & EAll cursos & 5 \\
\hline & & & & & & & AGRONEGOCIOS & 1 \\
\hline & & & & & & & EDUCACAO & 2 \\
\hline & & & & & & & DNFORMATICA & 2 \\
\hline & \multirow[t]{4}{*}{ SOLTEIRO } & \multirow[t]{4}{*}{ - All portador_dplomas } & \multirow[t]{4}{*}{${ }^{-}$All publica_particulars } & \multirow[t]{4}{*}{ EAll stuacao_cursos } & \multirow[t]{4}{*}{ †All forma_entroda_cursos } & \multirow[t]{4}{*}{ - ${ }_{\text {All ano_entradas }}$} & EAll cursos & 114 \\
\hline & & & & & & & AGRONEGOCIOS & 46 \\
\hline & & & & & & & EDUCACAO & 11 \\
\hline & & & & & & & DFFORMATICA & 57 \\
\hline
\end{tabular}

A consulta da figura 3 apresenta outro exemplo possível de consulta Olap, a qual mostra a quantidade de estudantes por estado civil. A figura 4 mostra a quantidade de estudantes por situação no curso, podendo ser em andamento, concluído e outro, sendo esta os desistentes ou em situação de trancamento.

Regae: Rev. Gest. Aval. Educ. 
Figura 4 -

Consulta Olap Analyticpos - situação no curso.

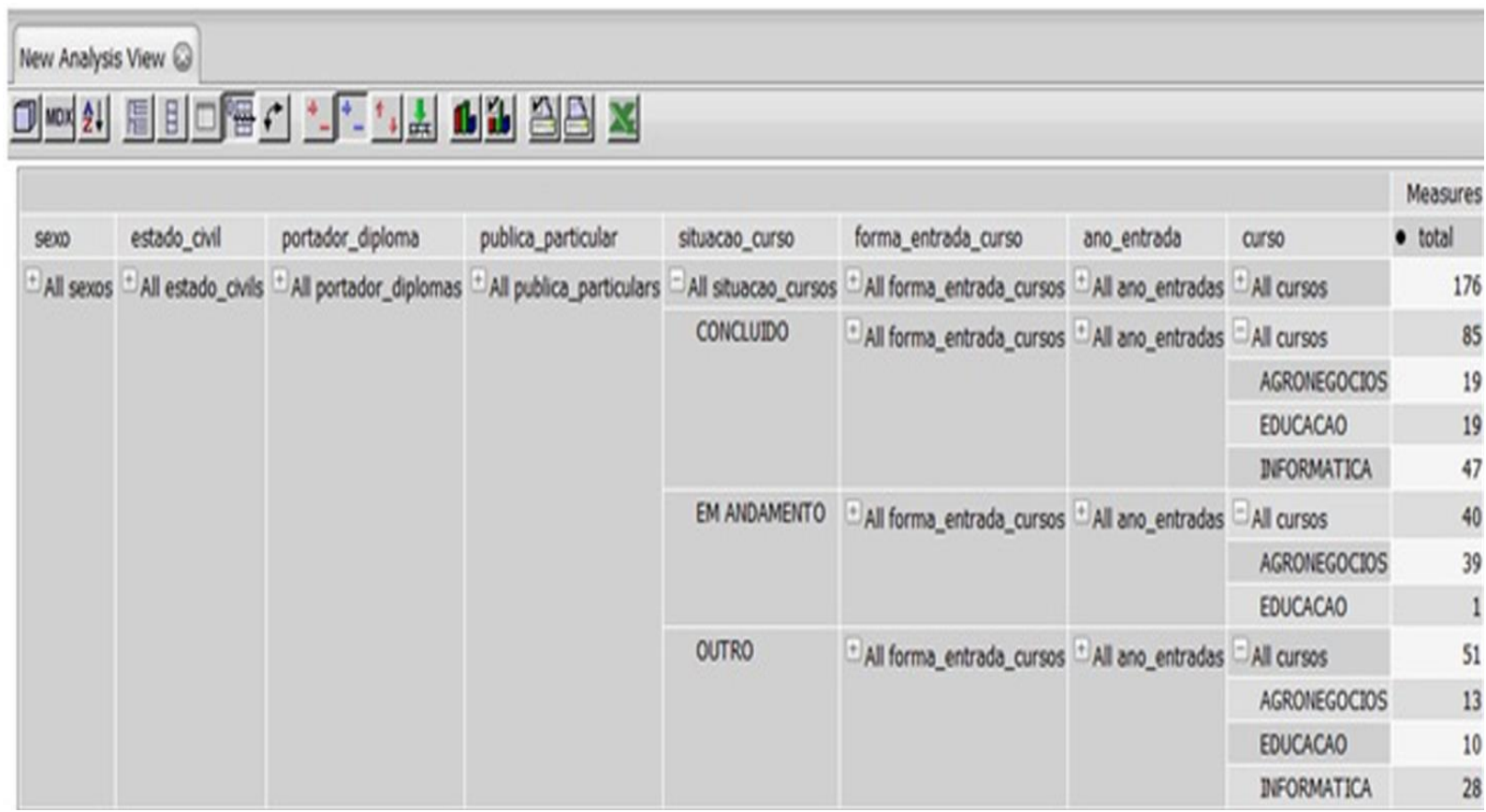

Pelas consultas realizadas na Analyticpos pode-se obter as seguintes informações sobre o perfil acadêmico dos cursos de pós-graduação, considerando os dados de todas as turmas existentes até o presente momento:

a) aproximadamente $65 \%$ dos estudantes são solteiros;

b) $48 \%$ dos estudantes encontram-se na situação concluído e, aproximadamente, $23 \%$ na situação em andamento, significando que, provavelmente, a porcentagem de desistência permaneça pequena se comparada com a situação outro atual;

c) $85 \%$ dos estudantes são provenientes de escolas privadas;

d) nenhum estudante é proveniente do IFC - campus de Videira, sendo que até o momento não tiveram formandos nos cursos de graduação do instituto;

e) todos os estudantes entraram no curso por edital.

A partir de consultas como estas é possível tomar algumas decisões como, por exemplo, a adoção de políticas para atrair os possíveis formandos dos cursos de graduação para cursarem a pós-graduação no próprio instituto.

Os resultados mostram que a permanência nos cursos é superior a $70 \%$. Os dados obtidos com as consultas podem ser utilizados, também, para orientar ações no sentido de envolver os estudantes em atividades na própria instituição, como bolsistas de projetos de ensino, pesquisa e extensão, com vistas a contribuir para a manutenção ou melhoria do índice de permanência no curso.

Outro fator que pode ser relacionado com o índice relativamente pequeno de evasão é a demanda que o município de Videira e região têm nas áreas dos cursos oferecidos ou, possivelmente, a maturidade dos estudantes na escolha do curso. 


\section{Conclusão e trabalhos futuros}

Com a criação da ferramenta é possível realizar consultas Olap na web sobre os dados dos estudantes dos cursos de pós-graduação do IFC - campus de Videira. A ferramenta oferece a possibilidade de analisar os dados por meio de uma interface amigável e de forma analítica, facilitando a tomada de decisão por parte dos coordenadores dos cursos.

No momento estão sendo aplicadas algumas técnicas de mineração de dados, como as regra de associação - algoritmo Tertius usando a ferramenta Weka (2015). Pelas regras de associação geradas será possível identificar e associar informações como, por exemplo, se os estudantes ingressaram em determinado ano a situação é em andamento ou concluído. Neste sentido, o presente projeto poderá auxiliar na identificação das causas que levam à desistência e propor políticas para diminuir a evasão ou estratégias para que os futuros concluintes nos cursos de graduação permaneçam no instituto.

Como trabalhos futuros poderão ser propostas pesquisas mais amplas sobre os estudantes, com vistas à melhorar e aumentar a utilidade da ferramenta. Além disso, poderá ser usado para os demais cursos presenciais do IFC e para ambas as modalidades de ensino em outras instituições que tenham interesse.

\section{Referências}

CAMPELO, Alex de Lima; ROSSI, Leila Lisiane; Aragão, Alfredo Lanari de, PIVA, Luis Henrique, MOTTA, Fernando Maia da. DWSI: data webhouse para análise do perfil acadêmico do curso de Sistemas de Informação (UFMS/CPCX). ENCONTRO NACIONAL DE ESTUDANTES DA COMPUTAÇÃO, 27, 2009. Anais ... Curitiba: UFPR, 2009.

INMON, Wiley H. Building the data warehouse. New York: John Wiley \& Sons, 2002.

JAVA, Sun Microsystems. Disponível em <http://www.java.com>. Acesso em 8 set., 2015.

KIMBALL, Ralph. Data warehouse toolkit: o guia completo para modelagem multidimensional. Rio de Janeiro: Campus, 2002.

PENTAHO. Open source business intelligence. Disponível em <http://www.pentaho.com>. Acesso em 8 set., 2015.

POSTGRESQL. Disponível em <http://www.postgresql.org>. Acesso em 8 set., 2015.

ROSSI; Leila Lisiane; SENKO, Luiz Gustavo Moro; HUBLER, Lizete Camara; HEINECK,Tiago; ROSA; Angela Maria Crotti da. Ferrramenta analytic para avaliar o perfil acadêmico do curso de Pedagogia - IFC - Videira. Revista Pedagogia UFMT. Cuiabá: UFMT, 2015a. Disponível em <http://www.revistapedagogiaufmt.com>. Acesso em 8 set., 2015.

ROSSI, Leila Lisiane; SENKO, Luiz Gustavo Moro; HEINECK; Tiago, ROSA; Angela Maria Crotti da. Analytic: ferramenta web para análise do perfil acadêmico do curso de Ciência da Computação - IFC - Videira. CONGRESSO INTERNACIONAL DE EDUCAÇÃO CIENTÍFICA E TECNOLÓGICA, 3, 2015. Erechim: URI, 2015b.

TURINE; Marcelo A. Santos, VIEIRA; Cristiano Argemon. Implantação, melhoria, revitalização e acompanhamento dos serviços de software que suportam as ações da Universidade Aberta do Brasil (UAB) na UFMS. Projeto de pesquisa. Campo Grande: UFMS, 2006.

WEKA, Pentaho. Data mining. Disponível em <http://community.pentaho.com/projects/ data-mining >. Acesso em 8 set., 2015. 
Leila Lisiane Rossi é mestre em Ciência da Computação e professora de Informática do Instituto Federal Catarinense - IFC - campus de Videira.

Endereço: Rodovia SC 135, Km 125 - Bairro Campo Experimental - 89560-000 Videira - SC - Brasil.

E-mail: leila.rossi@ifc-videira.edu.br.

Luiz Gustavo Moro Senko é mestre em Ciência da Computação e professor de Informática do Instituto Federal Catarinense - IFC - campus de Brusque.

Endereço: Rua Hercílio Luz, 373 - Centro - Brusque - SC - Brasil.

E-mail: gustavo.senko@brusque.ifc.edu.br.

Wanderson Rigo é mestre em Ciência da Computação, professor e coordenador do Curso de Informática do Instituto Federal Catarinense - IFC - campus de Videira.

Endereço: Rodovia SC 135, Km 125 - Bairro Campo Experimental - 89560-000 Videira - SC - Brasil.

CEP:89560-000 - Fone - 49-3533-4900

E-mail: wanderson.rigo@ifc-videira.edu.br.

Jeferson José Schneider Boesing é estudante do curso de graduação em Ciência da Computação - IFC - campus de Videira e colaborador do projeto de pesquisa.

Endereço: Rodovia SC 135, Km 125 - Bairro Campo Experimental - 89560-000 Videira - SC - Brasil.

E-mail: jefersonboesing@yahoo.com.br.

Recebido em 5 de outubro de 2015.

Aceito em 9 de dezembro de 2015. 